\title{
Sample cartridge with built-in miniature molecule evaporator for in-situ measurement with a photoemission electron microscope
}

\author{
Mohamad-Assaad Mawass ${ }^{1,}$, Jens Niederhausen ${ }^{1}$, Michael Hengge ${ }^{1,3}$, Katherine A. Mazzio ${ }^{1}$ \\ Simone Raoux ${ }^{1,2}$ and Florian Kronast ${ }^{1 \dagger}$ \\ ${ }^{1}$ Helmholtz-Zentrum Berlin für Materialien und Energie GmbH, Albert-Einstein-Str. 15, 12489 \\ Berlin, Germany \\ ${ }^{2}$ Institute of Physics, Humboldt University Berlin, Newtonstr. 15, 12489 Berlin, Germany \\ ${ }^{3}$ Present address: Institut für Physik, Institut für Chemie \& IRIS Adlershof, Humboldt-Universität \\ zu Berlin, Brook-Taylor-Str. 6, 12489 Berlin, Germany
}

\begin{abstract}
We present a new sample holder that is compatible with Photoemission Electron Microscopes (PEEMs) and contains a molecule evaporator. With the integrated low cost evaporator, a local and controlled material deposition in clean ultra-high vacuum conditions can be achieved minimizing the contamination of the analysis chamber. Different molecule systems can easily be studied by exchanging the sample holder. This opens up new possibilities for in-situ investigation of thin film growth by means of spectromicroscopy and element-selective imaging at the nanometer scale. As an example of the performances of the setup, we present a study of the hybrid inorganic/organic system (HIOS) consisting of the organic acceptor molecule 2,2'-(perfluoronaphthalene-2,6diylidene)dimalononitrile (F6TCNNQ) and $\mathrm{ZnO}$, which is of great interest for novel HIOS-based optoelectronic devices. Here, the $\mathrm{ZnO}$ surface work function modification by F6TCNNQ adsorption is investigated in-situ in a spatially resolved manner. In addition, we employ PEEM to selectively probe the chemical state of F6TCNNQ molecules monolayer in contact with $\mathrm{ZnO}$ and those molecules located in multilayers such as 3D grown islands.
\end{abstract}

\footnotetext{
*Email: mohamad.mawass@helmholtz-berlin.de

† Email: Florian.Kronast@helmholtz-berlin.de
} 


\section{Introduction:}

During the last two decades, the growth of small organic molecules at the nanoscale has gained attention because many of them are excellent candidates for future use in electronics [1, 2]. An experimental study often consists in preparation of a thin molecular film and its analysis in clean ultra-high vacuum conditions. The conventional approach for this is (i) evaporating the molecules in the preparation chamber and (ii) subsequently transferring the sample to the analysis chamber. This two-step so-called in-system approach has several merits. Most importantly, it guarantees an analysis chamber with minimum contaminants and complexity. However, in many cases an in-situ growth is desired, in particular when analysis during growth is required to prevent or capture the influence of post-growth dynamics and/or efficient sample preparation needs to be assisted by precise, quick, and simple analysis-supported preparation to guarantee for efficient beamtime use at the synchrotron (beamtime allocated for the experiment is usually restricted).

An alternative method is to build a conventional effusion cell directly into the analyzing chamber [3-5]. However in PEEM this bares some disadvantages. Firstly, in a PEEM chamber the access to the sample is limited by the objective lens. The evaporator can be only mounted few tens of centimeters away from the sample surface at the measurement position in front of the objective lens, enabling for a material deposition under grazing incidence for a maximum of $20^{\circ}$ [3]. This usually leads to significant contamination of UHV chamber and objective lens during evaporation. Secondly, the number of available ports for mounting evaporators is very limited, which usually restricts the number of molecule systems that can be studied during one measurement campaign without venting the PEEM chamber. Finally it is important to minimize and direct the flow of molecules required for film preparation to reduce the risk of electric discharges during growth and allow for real time observations during thin film growth. Where typically 10 to $20 \mathrm{kV}$ is applied between the sample and the objective lens of the microscope in order to accelerate the photoemitted electrons.

This means a radically different approach is needed as a new concept to render in-situ imaging suitable for PEEM experiment. In this paper, we present a specially developed sample holder for an ELMITEC-PEEM containing a low cost (homemade) miniature evaporator providing almost zero contamination of the experimental chamber with a direct and efficient molecule deposition by having a direct access to the sample surface. This new sample holder allows for in-situ molecule evaporation and direct imaging while depositing, thereby greatly extending the possibilities of the microscope.

First experiments with the new sample holder were carried out at the Spin-resolved Photoelectron Emission Microscope (SPEEM) [6, 7] at the BESSY II synchrotron facility of the HelmholtzZentrum Berlin (HZB), investigating the hybrid inorganic/organic system (HIOS) consisting of

Zinc Oxide $(\mathrm{ZnO})$ and organic molecules of 2,2'-(perfluoronaphthalene-2,6diylidene)dimalononitrile (F6TCNNQ). This molecule has a low evaporation temperature ( 120 ${ }^{\circ} \mathrm{C}$ ) and thus can be readily sublimed with a moderate filament current, Ifil, making it ideally suited for a conservative first evaporation experiment. More importantly, the ZnO/F6TCNNQ system is 
of great interest for novel HIOS-based optoelectronic devices: the performance and efficiency of HIOS devices rely on the precise and deterministic control of their interface energy-level alignment [8]. Accordingly, the investigation of interface properties in inorganic/organic systems has recently become the focus of intense scientific interest. For example, it was demonstrated that the work function of $\mathrm{ZnO}$ can be tailored by depositing a very thin film of electron donor or acceptor molecules in the (sub)monolayer thickness regime [9]. This observed behavior is due to the fact that the charge rearrangement between molecular film and inorganic semiconductor gives rise to an electrostatic potential step across the interface [10,11]. Notably, the nature of the interfacial interaction in the case of the strong electron acceptor F6TCNNQ and $\mathrm{ZnO}$ is yet unknown. In the case of F6TCNNQ in contact with graphene, different binding energies were observed for the $\mathrm{N} 1 \mathrm{~s}$ core level when comparing (sub)monolayer coverage (representative of the molecules in contact with substrate) and multilayer grown molecules (including also neutral molecules) [12]. Conventional photoelectron spectroscopy has yielded no such difference for ZnO. However, the high aspect ratio of the $3 \mathrm{D}$ islands formed by the multilayer molecules results in them having only a small spectral weight in an area-averaged measurement mode, due to the lack of space resolution in such conventional technique. In addition, such a non-imaging technique cannot exclude onset of 3D island formation before completion of the first monolayer. Thus, PEEM-controlled preparation and analysis of the $\mathrm{ZnO} / \mathrm{F} 6 \mathrm{TCNNQ}$ interface is a key prerequisite for safely ruling out a significant $\mathrm{N}$ 1s binding energy difference between neutral F6TCNNQ molecules and those in contact with $\mathrm{ZnO}$. This goal is achieved by combining the spatial resolution and element selectivity with the real-time imaging advantages provided by PEEM.

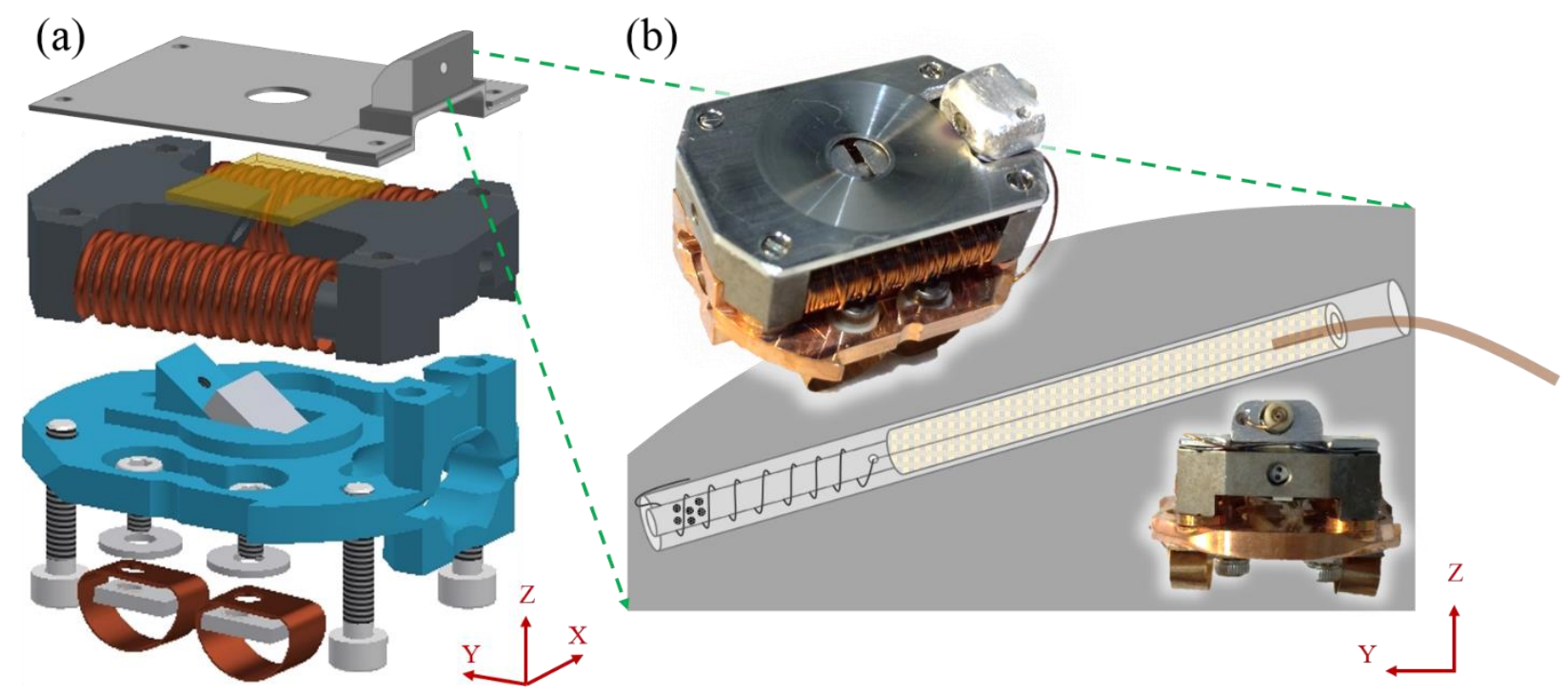

Figure 1: (a) A schematic drawing of a customized multifunctional sample holder with 4 electric contacts. Including an electromagnet with laser optics fixed below the sample position, temperature control and the tiny evaporator attached to the cap. (b) Photographs of the assembled sample holder is displayed in oblique and side view. A schematic drawing of the evaporator cross section, are shown as well, where the black small dots represents the materials to be deposited. 


\section{Experimental and design}

The sample holder with integrated evaporator is designed to be compatible with the commercial ELMITEC-PEEM III microscope which is widely used at various synchrotron sources [13]. At HZB a PEEM III with energy filter is permanently attached to an undulator beamline (UE49-PGMa at HZB) with full polarization control, in an energy range from 80 to 2,000 eV. The lateral resolution of the PEEM is about $25 \mathrm{~nm}$ for X-ray excitation [7].

A particular advantage of the ELMITEC system is its rather large sample cartridge supporting up to six electrical contacts. Many modifications have been reported enabling the injection of current pulse [14-16], electric [17-19] and magnetic field application [6, 20], temperature control [20] and even laser excitations [21]. The presented miniature molecule evaporator is compatible with most of the modified sample holders, it just requires one free pin to connect the heater. For example in Fig. 1 the evaporator is mounted on a sample holder with integrated laser optics, magnetic field and temperature control [21], allowing for multiple PEEM measurement environment at the same time. This evaporator can be built within very short time at virtually no cost.

Design and mounting of the molecule evaporator is shown in Fig. 1a. The evaporator itself consist of two small ceramic tubes (hollow cylinders) one inside another, separated by a glass fiber tube for thermal isolation. The heater is a tungsten filament $(0.125 \mathrm{~mm}$ diameter $)$ coming out from a small hole from the middle of the inner tube and wrapped in a solenoidal way up to its end, where a small quantity of molecules to be evaporated are placed, as illustrated in Fig. 1b. On that front end the heating filament is in touch with the body of the evaporator and on the back end it connects to a copper cable inserted from the back-side to the inner tube. The body of the evaporator is made of aluminum with rounded edges avoiding noticeable deformations of the extraction field by the objective lens. The evaporator is fixed into a boring within its aluminum body pointing towards the sample surface at an angle of $20^{\circ}$. A taper at the front end of the boring serves as the aperture and avoids that ceramic parts charge under exposure to UV or X-ray light. To minimize thermal crosstalk between sample and evaporator the body of the evaporator is supported by a thin metal sheet instead of attaching it directly of the cap of the sample holder.

To prepare the subsrate for the thin film deposition, the O-terminated side (000-1) of a hydrothermally grown ZnO crystal (CrysTec, Berlin) was cleaned in-situ by Ar+ sputtering (500 $\mathrm{eV}, 15$ minutes). We note that due to the low power of the used sample heating stage no annealing of the $\mathrm{ZnO}$ crystal was carried out. F6TCNNQ with an evaporation temperature is $\sim 120{ }^{\circ} \mathrm{C}$ was purchased from Novaled in Dresden.

\section{Results and discussion}

A careful characterization of the new setup during the molecules evaporation has been done. We carefully start to ramp the filament current and monitor the heating power. The molecular acceptor F6TCNNQ (electron affinity $=5.6 \mathrm{eV}$ [22]) was then deposited onto the $\mathrm{ZnO}$ surface for a few minutes. The onset of evaporation starts at a heating power of about $1.85 \mathrm{~W}$ and was determined by monitoring the intensity of secondary electrons emitted in UV-PEEM mode in real-time imaging. 
The employed UV lamp has a maximum photon energy of $\sim 4.9 \mathrm{eV}$, i.e. slightly higher than necessary to create photoelectrons that can overcome the $\mathrm{ZnO}$ work function, $\phi$, of ca. $4 \mathrm{eV}$. Therefore the F6TCNNQ adsorption increases the $\mathrm{ZnO}$ work function apparent by a darkening of the UV-PEEM image. The observed homogeneous darkening evidences a lack of 2D islands formation in the monolayer thickness regime. Thus, the deposition of the first monolayer of material is found to assemble without the formation of islands until full coverage (1 ML) is achieved. After each deposition cycle secondary electron cut-off (SECO) and X-ray photoelectron spectroscopy (XPS) (around the F 1s and N 1s core level) measurements, as function of the electron relative kinetic energy, are done by recording XPEEM images using the synchrotron radiation and turning off the UV lamp. Once the $\mathrm{ZnO}$ surface is covered with 1ML of F6TCNNQ, 3D islands start forming that can be readily identified as darker spots (see Figs. $2 \mathrm{a}$ and $3 \mathrm{a}$ ). This enable us to calculate the deposition rate, which was in our case, approximately, one monolayer per 10 min for $1.85 \mathrm{~W}$ heating power.

During evaporation the pressure increased about one order of magnitude from $3 \mathrm{e}-9$ mbar to $\sim 3 \mathrm{e}-8$ mbar. This order of magnitude is typical also during molecular depositions from a conventional evaporator. We want to point out that the pressure gauge only registers the degassing of the evaporator and its surrounding and not the molecular flux from the evaporator. This is because the molecules have a very high sticking coefficient at the chamber walls. Therefore, taking into account the evaporation geometry, the PEEM and other sensitive equipment will stay much cleaner with the micro evaporator.

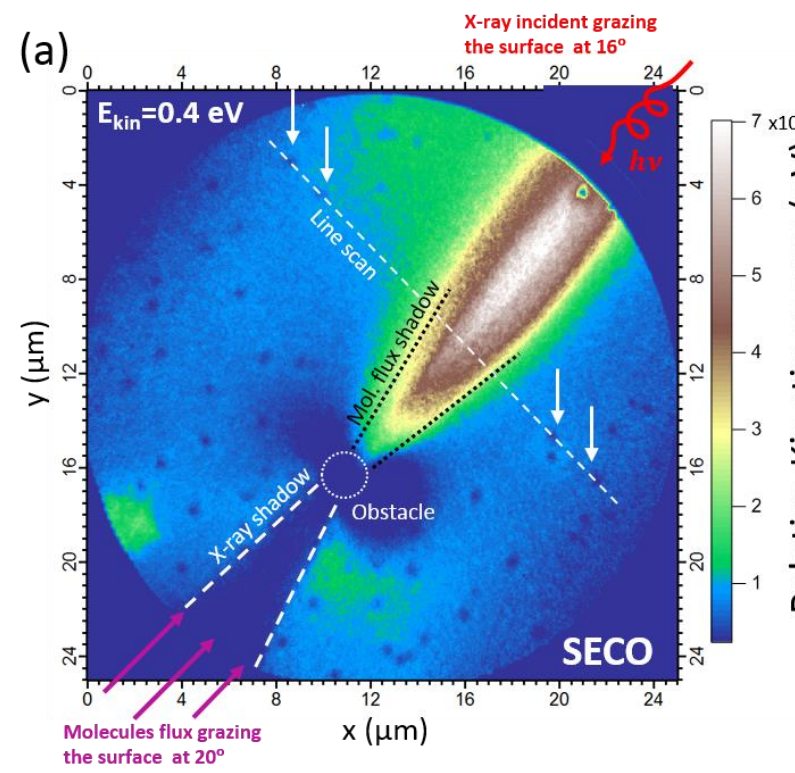

(b)

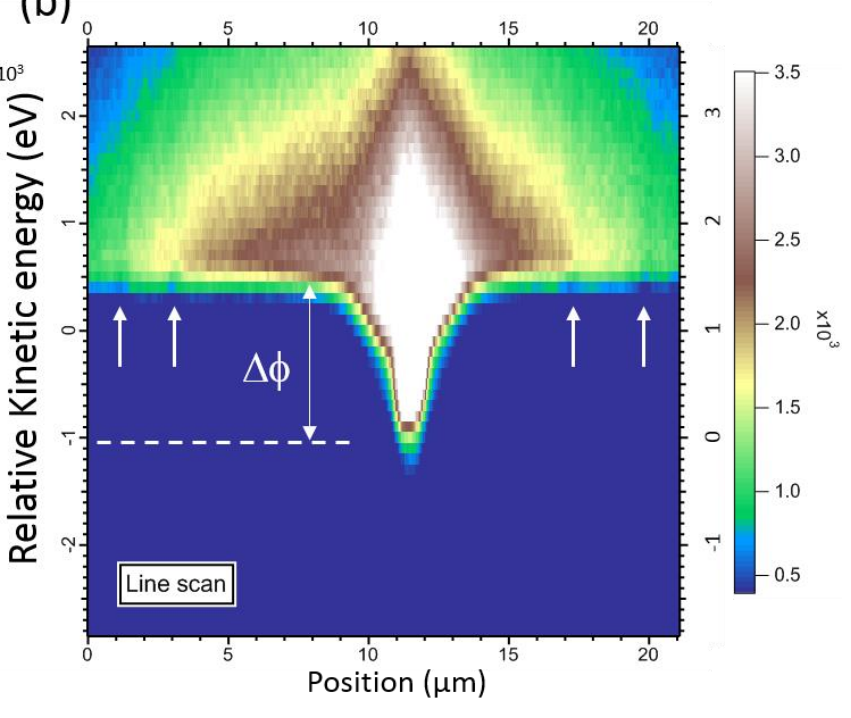

Figure 2: (a) spatially resolved XPEEM snapshot image extracted from the SECO measurement where $E_{\text {kin }}=0.4 \mathrm{eV}$. The image field of view is $25 \mu \mathrm{m}$. (b) Image showing the SECO image plot along the white dashed line indicated in (a). This line scan is crossing three different regions: (i) the deposited F6TCNNQ molecule monolayer region (the blue background). (ii) The 3D grown molecule islands (in dark blue dots). (iii) The molecule flux shadow region or the uncovered $\mathrm{ZnO}$ area. Extracting the relative kinetic energy across these regions allows us to derive $\Delta \phi$, where the dip represent the relative kinetic energy extracted from the uncovered $\mathrm{ZnO}$ are. The white arrows in (b) points to the dots marked with white arrows in (a). 
Thus, keeping the clean ultra-high vacuum conditions and allowing for a real-time imaging without turning off the objective high voltage or causing any static discharges of the sample, was possible. Furthermore, the sample temperature increased by only $\sim 10^{\circ}$, measured by the PT100 temperature sensor embedded in our customized sample holder allowing for a real-time temperature monitoring, This small increase of temperature indicates a working thermal isolation between sample and evaporator. This temperature increase does not have any influence on the physical properties of the system and can be neglected.

In principle the exact determination of the material coverage as a function of distance from the evaporator would be possible by locally resolving the onset of 3D island growth. The thickness coverage is expected to change, in our case, by $\sim 220 \%$ across the whole aperture of the sample holder $(\sim 3500 \mu \mathrm{m})$, therefore within the imaging field of view $(25 \mu \mathrm{m})$ the coverage would change by only $1.5 \%$ which can be neglected. Thus, one can consider the thickness homogeneous of the deposited material across the probed area.

The described real-time monitoring of the growth of the molecular film significantly eased the preparation of a film that exhibited 3D islands of moderate height required for the subsequent analysis described below. Area-integrated PEEM measurements, employing an excitation energy of $1486.6 \mathrm{eV}$, were used to determine the area ratio of the $\mathrm{C} 1 \mathrm{~s}$ and $\mathrm{O} 1 \mathrm{~s}$ core level spectra (not shown). This value was then compared with the $\mathrm{C} 1 \mathrm{~s}$ to $\mathrm{O} 1 \mathrm{~s}$ ratios obtained from previous experiments, wherein F6TCNNQ films of increasing thickness were stepwise deposited onto O$\mathrm{ZnO}$ [23]. These experiments were performed in a different setup and included ultraviolet photoelectron spectroscopy (UPS) measurements using HeI radiation in addition to XPS measurements using $\mathrm{Al} \mathrm{K} \alpha$ radiation. In this case, a conventional evaporator was employed and a quartz-crystal micro-balance allowed for an independent coverage determination. This calibration procedure via the $\mathrm{C} 1 \mathrm{~s}$ to $\mathrm{O} 1 \mathrm{~s}$ ratio yielded an approximate nominal F6TCNNQ thickness of $15 \AA$ for the film presented in Figs. 2 and 3. This is in line with an atomic force microscopy study of the sample after it had been taken out of the UHV system that found 3D islands of 50-150 nm height and an average film thickness of ca. $16 \AA$.

Organic molecules are known to be prone to radiation damage. We therefore carefully assessed the X-ray-induced degradation of F6TCNNQ by monitoring concomitant changes in the corresponding core level spectra. Based on this analysis, which can be found in the supplementary information, we detuned the undulator and thereby decreased the X-ray intensity by more than one order of magnitude. The data presented in the main manuscript are representative of the pristine molecular film and were exclusively obtained for spots that had not been previously exposed to X-rays.

Thanks to the grazing incidence geometry of the molecular flux, even small protrusions give rise to substantial shadowed areas in the PEEM field of view. Since the evaporator opening, while macroscopically small, is still large compared to these microscopic protrusions, the shadow of the molecular flux is not sharp but yields an in-plane coverage gradient behind the protrusions. An example for this can be seen in Fig. 2a. The bright area in the top right corner corresponds to a region that at its center is uncovered by F6TCNNQ molecules. Note that this area is opposite to the 
shadow of the X-ray, since the molecule flux direction and the X-ray photons incidence are opposite, as shown in Fig. 2a. The evaporator is located exactly downstream from the sample when looking along the beamline. As can be seen when following the line scan in Fig. $2 b$ from the uncovered center left- or rightwards, $\Delta \phi$, apparent from the secondary electron cut-off (SECO), gradually increases until it saturates at a constant value once the position reaches the monolayer covered part of the sample. The line scan yields an additional slight increase in $\Delta \phi$ for the 3D islands (marked by the arrows in Fig. 2). These observations are consistent with the results from area-integrated UPS [23] and Kelvin probe force microscopy [24].

Figure 3 shows another region for the same sample where another protrusion casts a characteristic $\mathrm{X}$-ray shadow. The excitation photon energy used here is $470 \mathrm{eV}$. The shadow was used as reference in order to compensate for small differences in the imaging by the electronic lens system when scanning the secondary and the photoelectrons around the $\mathrm{N} 1 \mathrm{~s}$ core level energetic region (the images are slightly rotated and shifted with respect to each other). This then allowed to compare the $\mathrm{N}$ 1s spectra for F6TCNNQ molecules interacting with the $\mathrm{ZnO}$ and those that are in the 3D islands and thus are representative for bulk F6TCNNQ. No difference in energetic position can be seen, despite the strong interaction at the ZnO/F6TCNNQ interface apparent from the large work function increase, $\Delta \phi$, shown in Fig. $2 b$.
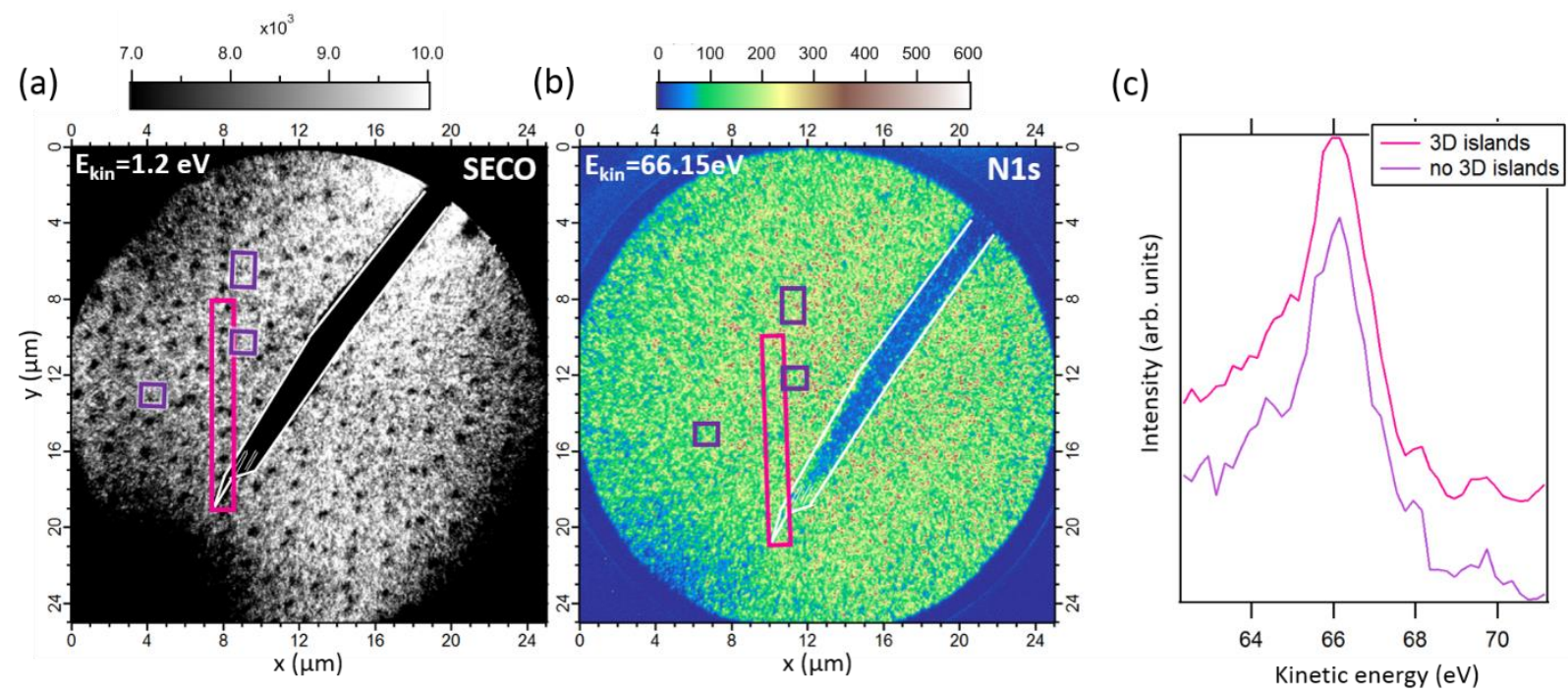

Figure 3: (a) and (b) XPEEM snapshot images of the identical region extracted from the SECO measurement (at $E_{\text {kin }}=1.2 \mathrm{eV}$ ) and the energy scan across the $\mathrm{N} 1 \mathrm{~s}$ core level (at $\mathrm{E}_{\mathrm{kin}}=66.15 \mathrm{eV}$ ) shown in different color scale for better illustration, respectively. The images field of view is 25 $\mu \mathrm{m}$. The photon energy, $\boldsymbol{h v}=\mathbf{4 7 0} \boldsymbol{e . V}$ (c) spatially resolved XPS spectra of the $\mathrm{N}$ 1s core level extracted from the region of interest (ROIs) indicated by pink and purple rectangles which is dominated by regions with a F6TCNNQ monolayer and 3D islands, respectively, shown in (a) and (b). 


\section{Conclusions}

In summary, we have presented a new sample holder compatible with ELMITEC PEEMIII containing a low cost tiny evaporator. This new sample holder allows for in-situ molecule evaporation and direct imaging during the deposition, which greatly extends the possibilities of the microscope. The developed setup is highly flexible and compatible with different customized ELMITEC sample holders in use at BESSY II PEEM experimental station at the SPEEM beamline. Furthermore, the evaporated material can be easily replaced by removing the sample holder from the vacuum chamber instead of venting the system to exchange materials to be deposited in an evaporator, providing the necessary new concept to render in-situ imaging suitable and more convenient for PEEM experiments.

We have demonstrated the capabilities of the setup by investigating the hybrid inorganic/organic system consisting of F6TCNNQ molecules and $\mathrm{ZnO}$, which is of great interest for novel HIOSbased optoelectronic devices. We could successfully demonstrate a controlled and efficient molecule deposition in clean ultra-high vacuum conditions with almost no contamination of the experimental chamber.

Thanks to the real-time imaging employing the UV lamp, we could efficiently grow a film of the desired morphology. We could then selectively probe F6TCNNQ molecules located in 3D islands or areas where monolayer molecules are in direct contact with $\mathrm{ZnO}$. The grazing incidence geometry of the molecular flux gives rise to substantial shadowed areas in the PEEM field of view. These shadows are non-sharp because of the finite size of the evaporator outlet, resulting in coverage gradients behind the objects. We could show that when making use of this effect, a single sample spot can be exploited as convenient (quasi) growth study - from uncovered substrate to bulk-like film thickness. Specifically, we compared N 1s spectra for F6TCNNQ molecules in contact with $\mathrm{ZnO}$ and for those representative for bulk F6TCNNQ. Despite the strong interaction at the $\mathrm{ZnO} / \mathrm{F} 6 \mathrm{TCNNQ}$ interface apparent from the huge work function increase, even the high selectivity of our PEEM experiments yields no difference in energetic position.

The results suggest that PEEM together with the presented sample holder can give new insights into the field of the hybrid inorganic/organic systems or other in-situ measurements in combination with different required measurement environments. This opens up new possibilities for investigations of thin film growth with in-situ high spatial resolution measurements using the PEEM microscopes. Finally, it is worth mentioning that a second miniature evaporator could also be implemented for more complex experiments and when more than one materials is needed at the same time.

\section{Acknowledgments}

We thank HZB for the allocation of synchrotron radiation beamtime. We thank the Norbert Koch group of the Humboldt University of Berlin for providing the F6TCNNQ. 


\section{References}

[1] F. Schreiber. Organic molecular beam deposition: Growth studies beyond the first monolayer. Phys. Status Solidi A 201, 1037-1054 (2004).

[2] D. Gatteschi, L. Bogani, A. Cornia, M. Mannini, L. Sorace, R. Sessoli. Molecular magnetism, status and perspectives. Solid StateSci. 10, 1701-1709 (2008).

[3] H. Marchetto et al. Direct observation of epitaxial organic film growth: temperature-dependent growth mechanisms and metastability. Phys. Chem. Chem. Phys., 17, 29150-29160 (2015).

[4] A. J. Fleming, F. P. Netzer and M. G. Ramsey. Nucleation and 3D growth of para-sexiphenyl nanostructures from an oriented 2D liquid layer investigated by photoemission electron microscopy. J. Phys.: Condens. Matter 21, 445003 (2009).

[5] F.-J. Meyer zu Heringdorf. The application of low energy electron microscopy and photoemission electron microscopy to organic thin films. J. Phys.: Condens. Matter 20, 184007 (2008).

[6] F. Kronast, J. Schlichting, F. Radu, S. K. Mishra, T. Noll, and H. A. Dürr. Spin-resolved photoemission microscopy and magnetic imaging in applied magnetic fields. Surf. Interface Anal. 42, 1532-1536 (2010).

[7] F. Kronast, S. Valencia Molina. SPEEM: The photoemission microscope at the dedicated microfocus PGM beamline UE49-PGMa at BESSY II. Journal of large-scale research facilities, 2, A90. (2016).

[8] R. Schlesinger et al. Efficient light emission from inorganic and organic semiconductor hybrid structures by energy-level tuning. Nat. Commun. 6:6754 (2015).

[9] T. Schultz et al. Tuning the work function of GaN with organic molecular acceptors. Phys. Rev. B 93, 125309 (2016).

[10] H. Kang, J.-H. Kim, J.-K. Kim, J. Seo, and Y. Park. Interface electronic structure of a strongly electron withdrawing molecule on an indium-tin-oxide surface. J. Korean Phys. Soc. 59, 3060 (2011).

[11] R. Schlesinger, Y. Xu, O. T. Hofmann, S. Winkler, J. Frisch, J. Niederhausen, A. Vollmer, S. Blumstengel, F. Henneberger, P. Rinke, M. Scheffler, and N. Koch, Phys. Rev. B 87, 155311 (2013).

[12] C. Christodoulou et al. Tuning the electronic structure of graphene by molecular dopants: Impact of the substrate. ACS Appl. Mater. Interfaces, 7, 19134-19144 (2015).

[13] ELMITEC Elektronenmikroskopie GmbH, http://www.elmitec-gmbh.com/.

[14] L. Heyne et al. In situ contacting and current-injection into samples in photoemission electron microscopes. Rev. Sci. Instrum. 81, 113707 (2010). 
[15] S. Finizio et al. Domain wall transformations and hopping in $\mathrm{La} 0.7 \mathrm{Sr} 0.3 \mathrm{MnO} 3$ nanostructures imaged with high resolution x-ray magnetic microscopy. J. Phys.: Condens. Matter 26456003 (2014).

[16] J. Miguel, M. Bernien, D. Bayer, J. Sánchez-Barriga, F. Kronast, M. Aeschlimann, H. A. Dürr, and W. Kuch. A new sample holder for laser-excited pump-probe magnetic measurements on a Focus photoelectron emission microscope. Rev. Sci. Instrum. 79, 033702 (2008).

[17] M. Buzzi, C. A. F. Vaz, J. Raabe, and F. Nolting. Electric field stimulation setup for photoemission electron microscopes. Rev. Sci. Instrum. 86, 083702 (2015).

[18] S. Finizio et al. ELECTRICAL-FIELD CONTROL OF MAGNETISM MEDIATED BY STRAIN IN Ni NANOSTRUCTURES FABRICATED ON PRE-POLED PMN-PT (011). SPINVol. 03, No. 03, 1340008 (2013).

[19] R. O. Cherifi et al. Electric-field control of magnetic order above room temperature. Nat. Mater. 13, 345-351 (2014).

[20] O. Sandig, J. Herrero-Albillos, F. M. Römer, N. Friedenberger, J. Kurde, T. Noll, M. Farle, and F. Kronast. Imaging magnetic responses of nanomagnets by XPEEM. J. Electron Spectrosc. Relat. Phenom. 185, 365-370 (2010).

[21] L. Gierster, L. Pape, A. A. Ünal, and F. Kronast. A sample holder with integrated laser optics for an ELMITEC photoemission electron microscope. Rev. Sci. Instrum. 86, 023702 (2015).

[22] F. Zhang and A. Kahn. Investigation of the High Electron Affinity Molecular Dopant F6TCNNQ for Hole-Transport Materials. Adv. Funct. Mater. 28, 1703780 (2018).

[23] T. Schultz et al. Impact of surface states and bulk doping level on hybrid inorganic/organic semiconductor interface energy levels J. Appl. Phys. 123, 245501 (2018)

[24] T. Meisel et al. Fingerprint of Charge Redistribution in the Optical Spectra of Hybrid Inorganic/Organic Semiconductor Interfaces J. Phys. Chem. C 122, 12913-12919 (2018). 